\title{
WEAK MIXING FOR NONSINGULAR BERNOULLI ACTIONS OF COUNTABLE AMENABLE GROUPS
}

\author{
Alexandre I. Danilenko
}

\begin{abstract}
Let $G$ be an amenable discrete countable infinite group, $A$ a finite set, and $\left(\mu_{g}\right)_{g \in G}$ a family of probability measures on $A$ such that $\inf _{g \in G} \min _{a \in A} \mu_{g}(a)>$ 0 . It is shown (among other results) that if the Bernoulli shiftwise action of $G$ on the infinite product space $\bigotimes_{g \in G}\left(A, \mu_{g}\right)$ is nonsingular and conservative then it is weakly mixing. This answers in positive a question by Z. Kosloff who proved recently that the conservative Bernoulli $\mathbb{Z}^{d}$-actions are ergodic. As a byproduct, we prove a weak version of the pointwise ratio ergodic theorem for nonsingular actions of $G$.
\end{abstract}

\section{INTRODUCTION}

Let $G$ be an amenable discrete infinite countable group. We call a nonsingular $G$ action $Q=\left(Q_{g}\right)_{g \in G}$ on a $\sigma$-finite measure space weakly mixing (see [GlWe], [DaSi] and references therein) if for each ergodic measure preserving $G$-action $R=\left(R_{g}\right)_{g \in G}$ on a standard probability space, the product $G$-action $\left(Q_{g} \times R_{g}\right)_{g \in G}$ is ergodic. Of course, every weakly mixing action is ergodic.

Given a countable set $A$ and a family $\left(\mu_{g}\right)_{g \in G}$ of probability measures on $A$, we set $X:=A^{G}$ and $\mu:=\bigotimes_{g \in G} \mu_{g}$. Throughout this section we denote by $T=$ $\left(T_{g}\right)_{g \in G}$ the left shiftwise action of $G$ on $X$, i.e. $\left(T_{g} x\right)_{h}:=x_{g^{-1} h}$ for all $x=$ $\left(x_{h}\right)_{h \in G} \in X$ and $g, h \in G$. The dynamical system $(X, \mu, T)$ is called a nonsingular Bernoulli $G$-action if $\mu \circ T_{g} \sim \mu$ for all $g \in G$. If $\mu_{g}=\mu_{h}$ for all $g, h \in G$ then $T$ preserves $\mu$ and the dynamical properties of probability preserving Bernoulli actions are well understood (see [OrWe]).

On the other hand, the purely nonsingular case is considerably less studied even for $G=\mathbb{Z}$. Some of nonsingular Bernoulli shifts can be nonconservative (see examples in [Ha] and [DaLe]). Krengel constructed in $[\mathrm{Kr}]$ the first conservative nonsingular Bernoulli shift which does not admit an equivalent invariant probability measure (see also [Ha] and [Ko1] for further refinements of his result). It was assumed in all those papers that $\mu$ is semistationary, i.e. there is $n \in \mathbb{Z}$ such that either $\mu_{n}=\mu_{n-1}=\mu_{n-2}=\cdots$ or $\mu_{n}=\mu_{n+1}=\mu_{n+2}=\cdots$. In the recent works [Ko3] and [DaLe], the semistationary nonsingular Bernoulli shifts were studied in depth in the framework of theory of nonsingular endomorphisms. In particular, it was shown that every such shift is either dissipative or weakly mixing, it possesses the nonsingular property $K$ (in the sense of [SiTh]) and Krieger's type of it (in the conservative case) is either $I I_{1}$ or $I I I_{1}$. A number of explicit examples of type $I I I_{1}$ Bernoulli transformations with various weak mixing properties were constructed by Vaes and Wahl in [VaWa]. They also showed that each infinite countable amenable group (in fact, a group from a much larger class of countable groups with nontrivial first $L^{2}$-cohomology) has Bernoulli actions of type $I I I_{1}$. 
Unfortunately, the aforementioned approach to semistationary shifts ( $\mathbb{Z}$-actions) does not work with the shifts $T$ which do not have an equivalent semistationary measure. Such shifts remain almost unstudied so far. It is known that they are of zero type [Ko2]. Some progress was achieved recently by Kosloff [Ko4] who proved that

(o) if $A$ is finite, $\inf _{n \in \mathbb{Z}} \min _{a \in A} \mu_{n}(a)>0$ and $T$ is conservative then $T$ is ergodic.

We refine and extend this as follows:

Theorem 0.1 (see Corollary 3.2, Theorem 3.5 below).

(i) For a finite $A$, if $\inf _{n \in \mathbb{N}} \min _{a \in A} \mu_{n}(a)>0$ and $T$ is conservative then $T$ is weakly mixing.

(ii) If $\# A=2, \inf _{n \in \mathbb{N}} \min _{a \in A}\left|\log \left(\frac{\mu_{n}(a)}{\mu_{n+1}(a)}\right)\right|<\infty$ and $T$ is conservative then $T$ is weakly mixing.

(iii) Under condition $(i)$ or $($ ii $)$, if $T \times \cdots \times T$ ( $p$ times $)$ is conservative for some $p>1$ then $T \times \cdots \times T(p$ times $)$ is weakly mixing.

We note that the condition on $\left(\mu_{n}\right)_{n=1}^{\infty}$ in $(i i)$ is weaker than the condition on $\left(\mu_{n}\right)_{n=1}^{\infty}$ in $(i)$. Hence $(i)$ and (o) follow from $(i i)$ in case where $\# A=2$. We also construct an explicit example of a weakly mixing nonsingular Bernoulli shift whose quasiinvariant measure is not equivalent to any semistationary one (see Example 3.3 below).

The proof of (o) in [Ko4] is based heavily on application of the Hurewicz nonsingular ergodic theorem and the maximal inequality. That proof is valid also for the nonsingular Bernoulli actions of $\mathbb{Z}^{d}, d<\infty$, and, apparently, some Heisenberg groups, however it does not extend to actions of groups for which the Hurewicz theorem fails, say $\bigoplus_{n=1}^{\infty} \mathbb{Z}$ or $\mathbb{Q}$ (see a discussion in [Ho]). In view of that Kosloff asks [Ko4, Problem from $\S 5.3]$ :

does (o) extend to nonsingular Bernoulli actions of arbitrary countable amenable group actions?

We answer in positive by demonstrating a stronger theorem.

Theorem 0.2 (see Corollary 2.4 below). Let $A$ be finite and let $G$ be an amenable discrete countable infinite group.

(i) If $\inf _{g \in G} \min _{a \in A} \mu_{g}(a)>0$ and $T=\left(T_{g}\right)_{g \in G}$ is conservative then $T$ is weakly mixing.

(ii) Under the condition of $(i)$, if the "diagonal" $G$-action $T \times \cdots \times T(p$ times $)$ is conservative for some $p>1$ then it is weakly mixing.

In fact, we deduce Theorem 0.2 from the following more general result (cf. [Ko4, Theorem 2]).

Theorem 0.3 (see Theorem 2.3 below). Let $(X, d)$ be a Polish ultrametric space, $\mathcal{R}$ a countable Borel equivalence relation on $X, \mu$ a probability Borel measure on $X$ and $Q=\left(Q_{g}\right)_{g \in G}$ a conservative nonsingular $G$-action on $(X, \mu)$. If there is a $\mu$-conull subset $X_{0} \subset X$ such that

- $\left(Q_{g} \times Q_{g}\right)\left(\mathcal{R} \cap\left(X_{0} \times X_{0}\right)\right)=\mathcal{R} \cap\left(X_{0} \times X_{0}\right)$ for all $g \in G$,

- if $\left(Q_{g} x, x\right) \in \mathcal{R}$ for some $x \in X_{0}$ and $g \in G$ then $g=1$,

- the restriction of $Q$ to the $\sigma$-algebra $\operatorname{Inv}(\mathcal{R})$ of $\mathcal{R}$-saturated subsets is ergodic, 
- $\lim _{g \rightarrow \infty} d\left(Q_{g} x, Q_{g} y\right)=0$ for all $(x, y) \in \mathcal{R} \cap\left(X_{0} \times X_{0}\right)$,

- there is a Borel map $\alpha: \mathcal{R} \rightarrow[1,+\infty)$ with

$$
\alpha(x, y)^{-1}<\frac{d \mu \circ Q_{g}}{d \mu}(x) / \frac{d \mu \circ Q_{g}}{d \mu}(y)<\alpha(x, y)
$$

for all $(x, y) \in \mathcal{R} \cap\left(X_{0} \times X_{0}\right)$ and each $g \in G$.

then $Q$ is ergodic. If, moreover, $\operatorname{Inv}(\mathcal{R})$ is trivial $(\bmod \mu)$ then $Q$ is weakly mixing.

In contrast to [Ko4], we do not use any form of the maximal inequality in the proof of Theorem 0.3. Our main tools are the measurable orbit theory and the following version of the pointwise ratio ergodic theorem for nonsingular group actions which is of independent interest.

Theorem 0.4 (see Appendix). Let $G$ be amenable. Then for each nonsingular action $T=\left(T_{g}\right)_{g \in G}$ on a standard probability space $(X, \mu)$ and a countable subset $\mathcal{L}$ of $L^{1}(X, \mu)$, there is a Følner sequence $\left(F_{n}\right)_{n=1}^{\infty}$ in $G$ such that $\bigcup_{n=1}^{\infty} F_{n}=G$ and for each $f \in \mathcal{L}$, there exists

$$
\lim _{n \rightarrow \infty} \frac{\sum_{g \in F_{n}} f\left(T_{g} x\right) \frac{d \mu \circ T_{g}}{d \mu}(x)}{\sum_{g \in F_{n}} \frac{d \mu \circ T_{g}}{d \mu}(x)}=E(f \mid \mathfrak{T})(x)
$$

at a.e. $x$, where $\mathfrak{T}$ denotes the $\sigma$-algebra of $T$-invariant subsets in $X$.

Nonsingular Markov shifts were also considered in [Ko4]. It was shown there that under a natural boundedness condition, if the shifts are conservative then they are ergodic. We deduce from Theorem 0.3 that they are weakly mixing.

The outline of the paper is as follows. In $\S 1$ we briefly remind some basic concepts from the theory of measured equivalence relations. In $\S 2$ we prove Theorems 0.2 and 0.3. In the final $\S 3$ we consider the case $G=\mathbb{Z}$ in more detail. We first extend slightly Theorems 0.2 in this particular case (see Theorem 3.1) and then prove Theorem 0.1. The example of a conservative nonsingular Bernoulli shift whose quasiinvariant measure is not equivalent to semistationary one is also constructed there. A remark on nonsingular Markov shifts completes $\S 3$. Appendix contains a proof of Theorem 0.4.

Acknowledgements. I am grateful to M. Lemańczyk and S. Vaes for useful discussions and for finding some gaps in earlier versions of the paper. I also thank Z. Kosloff for useful remarks.

\section{Countable equivalence relations on measure spaces}

Let $(X, \mathfrak{B})$ be a standard Borel space. A Borel equivalence relation $\mathcal{R} \subset X \times X$ is called countable if for each $x \in X$, the $\mathcal{R}$-class $\mathcal{R}(x):=\{y \in X \mid(x, y) \in \mathcal{R}\}$ of $x$ is countable. If $\mathcal{R}(x)$ is finite for each $x$ then $\mathcal{R}$ is called finite.

Given a subset $B \in \mathfrak{B}$, we denote by $\mathcal{R}(B)$ the $\mathcal{R}$-saturation of $B$, i.e. $\mathcal{R}(B):=$ $\bigcup_{x \in B} \mathcal{R}(x)$. Of course, $\mathcal{R}(B) \in \mathfrak{B}$. A subset $B \in \mathfrak{B}$ is called $\mathcal{R}$-saturated (or $\mathcal{R}$-invariant) if $B=\mathcal{R}(B)$. We denote the collection of all $\mathcal{R}$-saturated subsets by $\operatorname{Inv}(\mathcal{R})$. It is a sub- $\sigma$-algebra of $\mathfrak{B}$. If $T$ is a Borel bijection $T$ of $X$ and $T \mathcal{R}(x)=\mathcal{R}(T x)$ at each $x \in X$, we say that $T$ normalizes $\mathcal{R}$. In this case the restriction of $T$ to $\operatorname{Inv}(\mathcal{R})$ is well defined. 
Let $A$ and $B$ be two countable sets, $\# A \geq 2$, $\# D=\infty$, and $X=A^{D}$. We define the tail equivalence relation on $X$ by setting that a point $x=\left(x_{d}\right)_{d \in D} \in X$ is equivalent to a point $y=\left(y_{d}\right)_{d \in D} \in X$ if there is a finite subset $D_{0} \subset D$ such that $x_{d}=y_{d}$ whenever $d \notin D_{0}$.

Let $\mu$ be a probability measure on $(X, \mathfrak{B})$. We say that $\mathcal{R}$ is $\mu$-nonsingular if $\mu(\mathcal{R}(B))=0$ whenever $\mu(B)=0$. If each subset from $\operatorname{Inv}(\mathcal{R})$ is either $\mu$-null or $\mu$-conull then $\mathcal{R}$ is called $\mu$-ergodic. If $\mathcal{R}(x) \cap B \neq \emptyset$ for each subset $B \in \mathfrak{B}$ for a.e. $x \in B$ then $\mathcal{R}$ is called $\mu$-conservative. If $\mathcal{R}$ is $\mu$-nonsingular and there is an increasing sequence $\mathcal{R}_{1} \subset \mathcal{R}_{2} \subset \cdots$ of finite equivalence relations $\mathcal{R}_{n}, n \in \mathbb{N}$, such that $\mathcal{R}(x)=\bigcup_{n=1}^{\infty} \mathcal{R}_{n}(x)$ for a.e. $x \in X$ then $\mathcal{R}$ is called $\mu$-hyperfinite and the sequence $\left(\mathcal{R}_{n}\right)_{n=1}^{\infty}$ is called a filtration of $\mathcal{R}$. A Borel equivalence subrelation of a $\mu$-nonsingular equivalence relation is $\mu$-nonsingular itself.

For each countable $\mathcal{R}$, there is a countable group $\Gamma$ of Borel isomorphisms of $X$ such that $\mathcal{R}$ is the orbit equivalence relation of $\Gamma$ [FeMo]. Such a group $\Gamma$ is not unique. However $\mathcal{R}$ is $\mu$-nonsingular, $\mu$-ergodic or $\mu$-conservative if and only if $\Gamma$ is $\mu$-nonsingular, $\mu$-ergodic or $\mu$-conservative respectively.

If $\mathcal{R}$ is $\mu$-nonsingular then there exists a Borel map $\Delta_{\mathcal{R}, \mu}: \mathcal{R} \rightarrow \mathbb{R}_{+}^{*}$ such that

$$
\Delta_{\mathcal{R}, \mu}(x, y)=\Delta_{\mathcal{R}, \mu}(y, z) \Delta_{\mathcal{R}, \mu}(z, x) \quad \text { for all }(x, y),(y, z) \in \mathcal{R}
$$

and $\Delta_{\mathcal{R}, \mu}(x, \gamma x)=\frac{d \mu \circ \gamma}{d \mu}(x)$ at a.e. $x$ for each $\mu$-nonsingular invertible Borel transformation $\gamma$ of $X$ with $(x, \gamma x) \in \mathcal{R}$ for a.e. $x$. We call $\Delta_{\mathcal{R}, \mu}$ the RadonNikodym cocycle of the pair $(\mathcal{R}, \mu)$. A $\mu$-nonsingular $\mathcal{R}$ is $\mu$-conservative if and only if $\sum_{y \in \mathcal{R}(x)} \Delta_{\mathcal{R}, \mu}(x, y)=+\infty$ at a.e. $x$. If $\mathcal{S}$ is a subrelation of $\mathcal{R}$ then $\Delta_{\mathcal{S}, \mu}=\Delta_{\mathcal{R}, \mu} \uparrow \mathcal{S}$. If $\mathcal{R}$ is finite and $f \in L^{1}(X, \mu)$ then the mathematical expectation of $f$ with respect to $\operatorname{Inv}(\mathcal{R})$ at a point $x \in X$ is the ratio

$$
\frac{\sum_{y \in \mathcal{R}(x)} f(y) \Delta_{\mathcal{R}, \mu}(x, y)}{\sum_{y \in \mathcal{R}(x)} \Delta_{\mathcal{R}, \mu}(x, y)} .
$$

For more information about measured equivalence relations (and measurable orbit theory) we refer to [DaSi] and references therein.

\section{Proof of Theorems 0.3 And 0.2 .}

Let $X$ be a Polish 0-dimensional space, $d$ an ultrametric on $X$ compatible with the topology, and $\mathcal{R}$ a countable Borel equivalence relation on $X$. Let $G$ be an amenable discrete infinite countable group and let $T=\left(T_{g}\right)_{g \in G}$ be a Borel free action of $G$ on $X$ that normalizes $\mathcal{R}$, i.e. $T_{g}$ normalizes $\mathcal{R}$ for each $g \in G$. We recall that given a probability measure $\mu$ on $X, T$ is called strictly $\mathcal{R}$-outer (mod $\mu$ ), if $T_{g} x \notin \mathcal{R}(x)$ for each $g \in G \backslash\{1\}$ at a.e. $x \in X$. Of course, a strictly $\mathcal{R}$-outer $G$-action is free $(\bmod \mu)$.

\section{Definition 2.1.}

(i) We say that $T$ squashes $\mathcal{R}$ if there is a $\mu$-conull subset $X_{0} \subset X$ such that $\lim _{g \rightarrow \infty} d\left(T_{g} x, T_{g} y\right)=0$ for each $(x, y) \in \mathcal{R} \cap\left(X_{0} \times X_{0}\right)$.

(ii) We say that $T$ is $\mathcal{R}$-bounded if there are a Borel map $\alpha: \mathcal{R} \rightarrow[1,+\infty)$ and a $\mu$-conull subset $X_{0} \subset X$ such that

$$
\alpha(x, y)^{-1} \leq \frac{d \mu \circ T_{g}}{d \mu}(x) / \frac{d \mu \circ T_{g}}{d \mu}(y) \leq \alpha(x, y)
$$

for all $(x, y) \in \mathcal{R} \cap\left(X_{0} \times X_{0}\right)$ and each $g \in G$. 
Remark 2.2. It is easy to verify that if $\mathcal{R}$ is $\mu$-nonsingular then $T$ is $\mathcal{R}$-bounded if and only if there are a Borel map $\beta: \mathcal{R} \rightarrow[1,+\infty)$ and a $\mu$-conull subset $Y_{0} \subset X$ such that

$$
\beta(x, y)^{-1} \leq \Delta_{\mathcal{R}, \mu}\left(T_{g} x, T_{g} y\right) \leq \beta(x, y)
$$

for all $(x, y) \in \mathcal{R} \cap\left(Y_{0} \times Y_{0}\right)$ and each $g \in G$.

We now state and prove the main result of this section.

Theorem 2.3. Let $\mu$ be a probability Borel measure on $X$ such that $T=\left(T_{g}\right)_{g \in G}$ is a conservative nonsingular $G$-action on $(X, \mu)$. Let $T$ normalize a countable Borel equivalence relation $\mathcal{R}$ on $X$ and let the restriction of $T$ to $\operatorname{Inv}(\mathcal{R})$ be $\mu$-ergodic ${ }^{1}$. If $T$ is strictly $\mathcal{R}$-outer, $\mathcal{R}$-bounded and squashes $\mathcal{R}$ then $T$ is ergodic. If, moreover, $\mathcal{R}$ is $\mu$-ergodic then $T$ is weakly mixing.

Proof. Suppose that $T$ is not ergodic. Denote by $\mathfrak{F}$ the $\sigma$-algebra of Borel $T$ invariant subsets. Then there is a subset $B \in \mathfrak{F}$ with $0<\mu(B)<1$. Let $E($. | $\mathfrak{F})$ stand for the mathematical expectation with respect to $\mathfrak{F}$. Select a sequence $\left(A_{n}\right)_{n=1}^{\infty}$ of open subsets in $X$ such that

$$
\frac{1}{n^{3}} \geq\left\|1_{B}-1_{A_{n}}\right\|_{1} \geq\left\|1_{B}-E\left(1_{A_{n}} \mid \mathfrak{F}\right)\right\|_{1}
$$

for each $n$. Then by the Markov inequality,

$$
\mu\left(\left\{x \in X|| 1_{B}(x)-E\left(1_{A_{n}} \mid \mathfrak{F}\right)(x) \mid>\frac{1}{n}\right\}\right) \leq \frac{1}{n^{2}}
$$

Hence the Borel-Cantelli lemma yields that for a.e. $x \in X$,

$$
\left|1_{B}(x)-E\left(1_{A_{n}} \mid \mathfrak{F}\right)(x)\right| \leq \frac{1}{n} \quad \text { eventually in } n
$$

Since $d$ is an ultrametric, we may assume without loss of generality that for each $n>0$, there is $\epsilon_{n}>0$ such that for each $x \in A_{n}$, the ball centered at $x$ and of radius $\epsilon_{n}$ is a subset of $A_{n}$. Denote by $\mathcal{T}$ the $T$-orbit equivalence relation on $(X, \mu)$. It is countable, $\mu$-nonsingular, $\mu$-conservative and $\mu$-hyperfinite. By Theorem A.1 (see Appendix below), there is an increasing sequence $H_{1} \subset H_{2} \subset \cdots$ of finite subsets in $G$ such that $\bigcup_{k=1}^{\infty} H_{k}=G$ and

$$
E\left(1_{A_{n}} \mid \mathfrak{F}\right)(x)=\lim _{k \rightarrow+\infty} \frac{\sum_{g \in H_{k}} 1_{A_{n}}\left(T_{g} x\right) \frac{d \mu \circ T_{g}}{d \mu}(x)}{\sum_{g \in H_{k}} \frac{d \mu \circ T_{g}}{d \mu}(x)}
$$

for each $n$ at a.e. $x \in X$. Since $T$ normalizes $\mathcal{R}$, it follows that $\mathcal{R}(B) \in \mathfrak{F}$. Therefore, by the condition of the theorem, $\mu(\mathcal{R}(B))=1$ and thus $\mu(\mathcal{R}(B) \backslash B)>0$. Hence there exist a Borel subset $B_{0} \subset B$, a $\mu$-nonsingular Borel one-to-one map $\gamma: B_{0} \rightarrow X \backslash B$ and $C>0$ such that $\mu\left(B_{0}\right)>0,(x, \gamma x) \in \mathcal{R}$ and $\alpha(x, \gamma x)<C$ for all $x \in B_{0}$. Since $T$ squashes $\mathcal{R}$, for a.e. $x \in X$ and each $n>0$, there is a finite subset $F_{n}(x)$ in $G$ such that $d\left(T_{g} x, T_{g} \gamma x\right)<\epsilon_{n}$ whenever $g \notin F_{n}(x)$. According to

\footnotetext{
${ }^{1}$ The latter is equivalent to the fact that the smallest equivalence relation containing $\mathcal{R}$ and the graphs of all $T_{g}, g \in G$, is $\mu$-ergodic.
} 
our choice of $\epsilon_{n}$, if $g \notin F_{n}(x)$ then $T_{g} x \in A_{n}$ if and only if $T_{g} \gamma x \in A_{n}$. Since $T$ is $\mathcal{R}$-bounded, we obtain that

$$
\frac{1}{\alpha(x, \gamma x)} \frac{d \mu \circ T_{g}}{d \mu}(\gamma x) \leq \frac{d \mu \circ T_{g}}{d \mu}(x) \leq \alpha(x, \gamma x) \frac{d \mu \circ T_{g}}{d \mu}(\gamma x)
$$

at a.e. $x \in B_{0}$. Since $T$ is conservative,

$$
\infty=\sum_{g \in G} \frac{d \mu \circ T_{g}}{d \mu}(x)=\lim _{k \rightarrow \infty} \sum_{g \in H_{k} \backslash F_{n}(x)} \frac{d \mu \circ T_{g}}{d \mu}(x)
$$

at a.e. $x$ and each $n>0$. Substituting (2-3) into (2-2) and using (2-4) we obtain that

$$
E\left(1_{A_{n}} \mid \mathfrak{F}\right)(x) \leq \alpha(x, \gamma x)^{2} E\left(1_{A_{n}} \mid \mathfrak{F}\right)(\gamma x) \leq C^{2} E\left(1_{A_{n}} \mid \mathfrak{F}\right)(\gamma x)
$$

for all $n>0$ and a.e. $x \in B_{0}$. Passing to the limit $(n \rightarrow \infty)$ and applying (2-2) we obtain that $1 \leq 0$, which is a nonsense. This proves the first claim of the theorem.

To prove the second one, take an ergodic probability preserving $G$-action $R=$ $\left(R_{g}\right)_{g \in G}$. Since every Borel $G$-action admits a countable generating partition [JaKeLo], we can realize $R$ as a left shiftwise action on the space $\mathbb{N}^{G}$ which is 0 -dimensional Polish in the usual infinite product topology. We define an ultrametric $d^{\prime}$ on $\mathbb{N}^{G}$ by setting $d^{\prime}(a, b):=\sum_{h \in G} \epsilon_{h}\left(1-\delta\left(a_{h}, b_{h}\right)\right)$, where $\delta(.,$.$) is the$ Kronecker delta, $\epsilon_{h}>0$ for each $h \in G$ and $\sum_{h \in G} \epsilon_{h}<\infty$. Denote by $\mathcal{T}$ the tail equivalence relation on $\mathbb{N}^{G}$. Then $R$ normalizes and squashes $\mathcal{T}$. The product space $X \times \mathbb{N}^{G}$ is 0 -dimentional Polish. Its topology is compatible with an ultrametric. The product $G$-action $T \times R:=\left(T_{g} \times R_{g}\right)_{g \in G}$ normalizes and squashes the product equivalence relation $\mathcal{R} \times \mathcal{T}$ on $X \times \mathbb{N}^{G}$. Moreover, $T \times R$ is strictly $(\mathcal{R} \times \mathcal{T})$-outer because $T$ is strictly $\mathcal{R}$-outer. Since

$$
\operatorname{Inv}(\mathcal{R} \times \mathcal{T})=\operatorname{Inv}(\mathcal{R}) \otimes \operatorname{Inv}(\mathcal{T})
$$

and $\operatorname{Inv}(\mathcal{R})$ is trivial $\bmod \mu$, the restriction of $T \times R$ to $\operatorname{Inv}(\mathcal{S} \times \mathcal{T})$ is isomorphic to the restriction of $R$ to $\operatorname{Inv}(\mathcal{T})$, which is ergodic because $R$ is ergodic on the entire Borel $\sigma$-algebra on $\mathbb{N}^{G}$. It is easy to verify that since $R$ is probability preserving, $T \times R$ is $(\mathcal{R} \times \mathcal{T})$-bounded. Since $T$ is conservative and $R$ is probability preserving, $T \times R$ is conservative. Thus we may apply the first claim of the theorem to conclude that $T \times R$ is ergodic. Hence $T$ is weakly mixing.

Given a countable set $A$, we let $X=A^{G}$ and denote by $\mathcal{T}$ the tail equivalence relation on $X$. Given a sequence $\left(\mu_{n}\right)_{n=1}^{\infty}$ of non-degenerated probability measures on $A$, we let $\mu:=\bigotimes_{n=1}^{\infty} \mu_{n}$. We will always assume that $\mu$ is non-atomic or, equivalently,

$$
\sum_{g \in G} \log \left(\max _{a \in A} \mu_{g}(a)\right)=-\infty .
$$

Then $\mathcal{T}$ is $\mu$-nonsingular and for all $\mathcal{T}$-equivalent points $x=\left(x_{h}\right)_{h \in G}$ and $y=$ $\left(y_{h}\right)_{y \in H}$ from $X$,

$$
\Delta_{\mathcal{T}, \mu}(x, y)=\prod_{h \in G} \frac{\mu_{h}\left(y_{h}\right)}{\mu_{h}\left(x_{h}\right)}
$$


We note that the product is finite indeed. It follows from the Kolmogorov 0-1 law that $\mathcal{T}$ is $\mu$-ergodic ${ }^{2}$. Let $T=\left(T_{g}\right)_{g \in G}$ denote the left shiftwise $G$-action on $X$. It follows from $[\mathrm{Ka}]$ that $T$ is nonsingular if and only if for each $g \in G$,

$$
\sum_{h \in G} \sum_{a \in A}\left(\sqrt{\mu_{h}(a)}-\sqrt{\mu_{g^{-1} h}(a)}\right)^{2}<\infty .
$$

Corollary 2.4. Let $(X, \mu, T)$ be a conservative nonsingular Bernoulli G-action with $A$ finite. If $\delta:=\inf _{g \in G} \min _{a \in A} \mu_{g}(a)>0$ then $T$ is weakly mixing. More generally, if the "diagonal" $G$-action $T \times \cdots \times T(p$ times $)$ is conservative for some $p>1$ then it is weakly mixing.

Proof. We note that $X$ is a Cantor (compact, ultrametric) space. Of course, $T$ normalizes and squashes $\mathcal{T}(\bmod \mu)$. Moreover, $T$ is strictly $\mathcal{R}$-outer $(\bmod \mu)$. Take $(x, y) \in \mathcal{T}$. Let $F_{x, y}:=\left\{g \in G \mid x_{g} \neq y_{g}\right\}$. It follows from (2-5) that

$$
\Delta_{\mathcal{T}, \mu}\left(T_{g} x, T_{g} y\right)=\prod_{h \in G} \frac{\mu_{g h}\left(y_{h}\right)}{\mu_{g h}\left(x_{h}\right)}=\prod_{h \in F_{x, y}} \frac{\mu_{g h}\left(y_{h}\right)}{\mu_{g h}\left(x_{h}\right)}
$$

Hence $\delta^{\# F_{x, y}} \leq \Delta_{\mathcal{T}, \mu}\left(T_{g} x, T_{g} y\right) \leq \delta^{-\# F_{x, y}}$. Thus, $T$ is $\mathcal{T}$-bounded by Remark 2.2. It now follows from Theorem 2.3 that $T$ is weakly mixing. Thus, the first claim is proved. The second claim follows from the first one.

\section{ON WEAK MIXING OF CONSERVATIVE TWO-SIDED SHIFTS}

In this section we consider in more detail the case where $G=\mathbb{Z}$. First we note that in this case the condition of the $\mathcal{R}$-boundedness in Theorem 2.3 can be slightly relaxed.

Theorem 3.1. Let $X$ be the same as in Theorem 2.2 and let $\mu$ be a probability Borel measure on $X$. Let $T$ be a conservative nonsingular transformation of $(X, \mu)$ that normalizes a countable Borel equivalence relation $\mathcal{R}$ on $X$ and let the restriction of $T$ to $\operatorname{Inv}(\mathcal{R})$ be $\mu$-ergodic. If $T$ is strictly $\mathcal{R}$-outer, squashes $\mathcal{R}$ and there is a Borel map $\alpha: \mathcal{R} \rightarrow[1,+\infty)$ and a $\mu$-conull Borel subset $X_{0} \subset X$ such that

$$
\alpha(x, y)^{-1}<\frac{d \mu \circ T^{-k}}{d \mu}(x) / \frac{d \mu \circ T^{-k}}{d \mu}(y)<\alpha(x, y)
$$

for all $(x, y) \in \mathcal{S} \cap\left(X_{0} \times X_{0}\right)$ and each $k \geq 0$ then $T$ is ergodic. If, moreover, $\mathcal{R}$ is $\mu$-ergodic then $T$ is weakly mixing.

Proof. The proof is almost a verbal repetition of the proof of Theorem 2.3. Only a slight modification is needed. Instead of (2-2), we should use the following limit

$$
E\left(1_{A_{n}} \mid \mathfrak{F}\right)(x)=\lim _{K \rightarrow+\infty} \frac{\sum_{k=0}^{K} 1_{A_{n}}\left(T^{-k} x\right) \frac{d \mu \circ T^{-k}}{d \mu}(x)}{\sum_{k=0}^{K} \frac{d \mu \circ T^{-k}}{d \mu}(x)}
$$

at a.e. $x \in X$ for each $n>0$. The existence of this limit follows from the Hurewicz ergodic theorem.

In a similar way one can "relax" the corresponding $\mathcal{R}$-boundedness condition in Remark 2.2.

We now discuss some applications of Theorem 3.1 to nonsingular Bernoulli shifts. The first claim of the following corollary strengthens [Ze, Theorem 3] (where it was proved that $T$ is ergodic under a stronger condition that $\left.\inf _{n \in \mathbb{Z}} \min _{a \in A} \mu_{n}(a)>0\right)$.

\footnotetext{
${ }^{2}$ Hint: enumerate the elements of $G$ with natural numbers.
} 
Corollary 3.2. Let $(X, \mu, T)$ be a conservative nonsingular Bernoulli $\mathbb{Z}$-shift with A finite. If $\delta:=\inf _{n>0} \min _{a \in A} \mu_{n}(a)>0$ then $T$ is weakly mixing. More generally, if the product $T \times \cdots \times T$ ( $p$ times $)$ is conservative for some $p>1$ then $T \times \cdots \times$ $T$ (p times) is weakly mixing.

Proof. Take $(x, y) \in \mathcal{T}$. Let $J_{x, y}:=\left\{k \in \mathbb{Z} \mid x_{k} \neq y_{k}\right\}$. It follows from (2-5) that

$$
\Delta_{\mathcal{T}, \mu}\left(T^{n} x, T^{n} y\right)=\prod_{k \in J_{x, y}} \frac{\mu_{k+n}\left(y_{k}\right)}{\mu_{k+n}\left(x_{k}\right)} .
$$

If $n$ is sufficiently large then $k+n>0$ for each $k \in J_{x, y}$. Hence we have eventually (in $n$ ) that $\delta^{\# J_{x, y}} \leq \Delta_{\mathcal{T}, \mu}\left(T^{n} x, T^{n} y\right) \leq \delta^{-\# J_{x, y}}$ It remains to apply Theorem 3.1 (and a corresponding analogue of Remark 2.2).

We now give some concrete examples of weakly mixing nonsingular Bernoulli shifts without an equivalent semistationary measure.

Example 3.3. Fix $\lambda \in(0,1 / 2)$ and a real $\xi$ such that

$$
\xi>\left(\frac{\lambda}{2}\right)^{-2}+\left(\frac{\lambda}{2}\right)^{-1}\left(1-\frac{\lambda}{2}\right)^{-2} .
$$

Choose a sequence of reals $\left(\alpha_{n}\right)_{n=1}^{\infty}$ and a sequence of positive integers $\left(A_{n}\right)_{n=0}^{\infty}$ such that the following conditions hold:

(i) $\alpha_{j} \in(0,1 / 2)$ and $\sum_{j=1}^{\infty} \alpha_{j}^{2}<+\infty$,

(ii) $A_{0}=0, A_{l}>8 A_{l-1}$ for each $l>0$ and

(iii) $\sum_{j=1}^{n} \alpha_{j}^{2} A_{j}=\frac{\log n}{4 \xi}$ for each $n>0$.

We now define for each $n \in \mathbb{Z}$, a measure $\mu_{n}$ on $A=\{0,1\}$ by setting

$$
\mu_{n}(0):= \begin{cases}\lambda, & \text { if } 2 A_{l} \leq|n|<A_{l+1} \text { for some } l \geq 0 \\ \lambda+\alpha_{l}, & \text { if } A_{l} \leq|n|<2 A_{l} \text { for some } l>0\end{cases}
$$

It is straightforward to verify that

(I) $\lambda / 2<\mu_{n}(0)<1-\lambda / 2$ for each $n \in \mathbb{Z}$ and

(II) $\sum_{n \in \mathbb{Z}}\left|\mu_{n+1}(0)-\mu_{n}(0)\right|^{2}=4 \sum_{j=1}^{\infty} \alpha_{j}^{2}<\infty$.

It follows from (I) that $\mu$ is non-atomic. It follows from (2-5) and (II) that $T$ is $\mu$-nonsingular. Of course, $\mu$ is not semi-stationary. It is straightforward to deduce from (I), (iii) and the Kakutani theorem [Ka] that $\mu$ is not equivalent to any semistationary measure on $X$. To show that $T$ is conservative we will apply [VaWa, Proposition 4.1], which states that if $\sum_{n \in \mathbb{Z}} e^{-\xi \sum_{j \in \mathbb{Z}}\left|\mu_{j+n}(0)-\mu_{j}(0)\right|^{2}}=+\infty$ then $T$ is conservative. Indeed, it follows from (ii) and (iii) that for each $l>0$,

$$
\sum_{j \in \mathbb{Z}}\left|\mu_{j-4 A_{l}}(0)-\mu_{j}(0)\right|^{2}=4 \sum_{j=1}^{l} \alpha_{j}^{2} A_{j}+4 \sum_{j>l} \alpha_{j}^{2} A_{l}=\frac{\log l}{\xi}+\bar{o}(1) .
$$

Then

$$
\begin{aligned}
\sum_{n \in \mathbb{Z}} e^{-\xi \sum_{j \in \mathbb{Z}}\left|\mu_{j+n}(0)-\mu_{j}(0)\right|^{2}} & \geq \sum_{l=1}^{+\infty} e^{-\xi \sum_{j \in \mathbb{Z}}\left|\mu_{j-4 A_{l}}(0)-\mu_{j}(0)\right|^{2}} \\
& =\sum_{l=1}^{+\infty} e^{-\log l+\bar{o}(1)}
\end{aligned}
$$


Hence $\sum_{n \in \mathbb{Z}} e^{-\xi \sum_{j \in \mathbb{Z}}\left|\mu_{j+n}(0)-\mu_{j}(0)\right|^{2}}=+\infty$ and thus $T$ is $\mu$-conservative. Corollary 3.2 yields that $T$ is weakly mixing.

We now investigate ergodicity of nonsingular Bernoulli shifts under assumption that $\delta=0$, where $\delta$ is defined in Corollary 3.2.

Let $\mathcal{S}$ be the symmetric equivalence relation on $X$. By definition, it is the orbit equivalence relation of the natural action of the group $\Sigma_{0}(\mathbb{Z})$ of all finite permutations of $\mathbb{Z}$ on $X$. In other words, two points $x=\left(x_{n}\right)_{n \in \mathbb{Z}}$ and $y=\left(y_{n}\right)_{n \in \mathbb{Z}}$ of $X$ are $\mathcal{S}$-equivalent if there is $\sigma \in \Sigma_{0}(\mathbb{Z})$ such that $x_{n}=y_{\sigma(n)}$ for all $n \in \mathbb{Z}$. It is easy to verify that $\mathcal{S}$ is a subrelation of $\mathcal{T}$ and $T$ normalizes $\mathcal{S}$. We will use the following result from $[\mathrm{AlPi}]$.

\section{Lemma 3.4.}

(i) Let $\# A=2$. Then $\mathcal{S}$ is $\mu$-ergodic if and only if $\sum_{n \in \mathbb{Z}} \min _{a \in A} \mu_{n}(a)=+\infty$, i.e. $\mu$ is non-atomic.

(ii) Let $A$ be finite. Then $\mathcal{S}$ is $\mu$-ergodic if and only if for each nonempty proper subset $B \subset A, \sum_{n \in \mathbb{Z}} \min \left(\mu_{n}(B), \mu_{n}(A \backslash B)=+\infty\right.$.

The following statement strengthens Corollary 3.2 in the case where $\# A=2$.

Theorem 3.5. Let $(X, \mu, T)$ be a nonsingular Bernoulli shift with $\# A=2$. If there is $D>1$ such that

$$
D^{-1}<\frac{\mu_{k}(a)}{\mu_{k+1}(a)}<D \quad \text { for all } a \in A \text { and each } k<0
$$

and $T$ is conservative then $T$ is weakly mixing. More generally, if $T \times \cdots \times$ $T$ ( $p$ times $)$ is conservative for some $p>1$ then $T \times \cdots \times T(p$ times $)$ is weakly mixing.

Proof. By Lemma 3.4(i), $\mathcal{S}$ is ergodic. Take $(x, y) \in \mathcal{S}$. Let $J_{x, y}:=\left\{k \in \mathbb{Z} \mid x_{k} \neq\right.$ $\left.y_{k}\right\}$ and let $\sigma$ be a permutation of $J_{x, y}$ such that $y_{k}=x_{\sigma(k)}$ for all $k \in J_{x, y}$. It follows from (2-5) that

$$
\Delta_{\mathcal{S}, \mu}\left(T^{n} x, T^{n} y\right)=\prod_{k \in J_{x, y}} \frac{\mu_{\sigma^{-1}(k)+n}\left(x_{k}\right)}{\mu_{k+n}\left(x_{k}\right)}
$$

If $n$ is large enough, we deduce from (3-1) that

$$
D^{-\left|\sigma^{-1}(k)-k\right|} \leq \frac{\mu_{\sigma^{-1}(k)+n}\left(x_{k}\right)}{\mu_{k+n}\left(x_{k}\right)} \leq D^{\left|\sigma^{-1}(k)-k\right|}
$$

for each $k \in J_{x, y}$. Since $\left|\sigma^{-1}(k)-k\right|<\# J_{x, y}$, we obtain that

$$
D^{-\left(\# J_{x, y}\right)^{2}} \leq \frac{\mu_{\sigma^{-1}(k)+n}\left(x_{k}\right)}{\mu_{k+n}\left(x_{k}\right)} \leq D^{\left(\# J_{x, y}\right)^{2}}
$$

eventually in $n$. Hence $T$ is weakly mixing by Theorem 3.1 and the analogue of Remark 2.2.

As in [Ko4], we note that Theorem 3.1 can be applied also to nonsingular Markov shifts. 
Remark 3.6. Let $S$ be a finite set and let $M=(M(a, b))_{a, b \in S}$ be a $\{0,1\}$-valued $(S \times S)$-matrix. Given two integers $i \leq j$ and a finite sequence $a=\left(a_{l}\right)_{i \leq l \leq j}$ of elements from $S$ such that $M\left(a_{l}, a_{l+1}\right)=1$ for each $l=i, \ldots, j-1$, we denote by $[a]_{i}^{j}$ the cylinder $[a]_{i}^{j}:=\left\{x \in X_{M} \mid x_{l}=a_{l}\right.$ for each $\left.l=i, \ldots, j\right\}$. The set of infinite paths $X_{M}:=\left\{x=\left(x_{i}\right)_{i \in \mathbb{Z}} \in S^{\mathbb{Z}} \mid M\left(x_{i}, x_{i+1}\right)>0\right\}$ is a closed subset of $S^{\mathbb{Z}}$ which is invariant under the two-sided shift $T$. Suppose that there is a sequence $\left(\pi_{n}\right)_{n \in \mathbb{Z}}$ of probability measures on $S$ and a sequence $\left(P_{n}\right)_{n \in \mathbb{Z}}$ of row-stochastic $(S \times S)$-matrices such that $\pi_{n} P_{n}=\pi_{n+1}$ and $P_{n}(a, b)>0$ if and only if $M(a, b)=1$ for each $n \in \mathbb{Z}$. Then there is a unique probability measure $\mu$ on $X_{M}$ such that for every cylinder $[a]_{i}^{j}$ in $X_{M}$,

$$
\mu\left([a]_{i}^{j}\right)=\pi_{i}\left(a_{i}\right) P_{i}\left(a_{i}, a_{i+1}\right) \cdots P_{j-1}\left(a_{j-1}, a_{j}\right)
$$

It is called a Markov measure on $X_{M}$ generated by $\left(\pi_{n}, P_{n}\right)_{n \in \mathbb{Z}}$. Denote by $\mathcal{T}$ the restriction of the tail equivalence relation to $X_{M}$. It is easy to verify that $\mathcal{T}$ is $\mu$-nonsingular and for each $(x, y) \in \mathcal{T}$,

$$
\Delta_{\mathcal{T}, \mu}(x, y):=\prod_{i \in \mathbb{Z}} \frac{P_{i}\left(y_{i}, y_{i+1}\right)}{P_{i}\left(x_{i}, x_{i+1}\right)}
$$

We note that the infinite product in this formula is indeed finite. Suppose that

- $M$ is primitive, i.e. there is $n>0$ with $M^{n}(a, b)>0$ for all $a, b \in S$ and

— that $\delta:=\inf \left\{P_{n}(a, b) \mid n \in \mathbb{Z}, M(a, b)=1\right\}>0$.

It was shown in [Ko4] that under these two conditions, $\mathcal{T}$ is $\mu$-ergodic. It follows from this fact, Theorem 3.1 and Remark 2.2 that if the shift $T$ on $\left(X_{M}, \mu\right)$ is conservative and nonsingular then $T$ weakly mixing. Indeed, it suffices to note that $\Delta_{\mathcal{T}, \mu}\left(T^{n} x, T^{n} y\right):=\prod_{i} \frac{P_{i+n}\left(y_{i}, y_{i+1}\right)}{P_{i+n}\left(x_{i}, x_{i+1}\right)}$ and argue as in Corollary 3.2.

\section{Appendix. WeAK POINTWise RATIO ERGODIC THEOREM FOR NONSINGULAR ACTIONS OF AMENABLE GROUPS}

We recall that given a finite subset $K \subset G$ and $\epsilon>0$, a finite subset $F \subset G$ is called $[K, \epsilon]$-invariant if $\#\{g \in F \mid K g \subset F\}>(1-\epsilon) \# F$.

Theorem A.1. Let $G$ be amenable. Fix a sequence $\left(K_{n}\right)_{n=1}^{\infty}$ of finite subsets in $G$ and a sequence of positive reals $\left(\epsilon_{n}\right)_{n=1}^{\infty}$ converging to 0 . Then for each nonsingular action $T=\left(T_{g}\right)_{g \in G}$ on a standard probability space $(X, \mu)$ and a countable subset $\mathcal{L}$ of $L^{1}(X, \mu)$, there is an increasing sequence $F_{1} \subset F_{2} \subset \cdots$ of finite subsets in $G$ such that $\bigcup_{n=1}^{\infty} F_{n}=G$ and for every $f \in \mathcal{L}$, there exists

$$
\lim _{n \rightarrow \infty} \frac{\sum_{g \in F_{n}} f\left(T_{g} x\right) \frac{d \mu \circ T_{g}}{d \mu}(x)}{\sum_{g \in F_{n}} \frac{d \mu \circ T_{g}}{d \mu}(x)}=E(f \mid \operatorname{Inv}(\mathcal{T}))(x)
$$

at a.e. $x$, where $\mathcal{T}$ denotes the $T$-orbit equivalence relation on $X$. Moreover, $F_{n}$ is $\left[K_{m}, \epsilon_{m}\right]$-invariant for each $m \leq n$. In particularly, if $\bigcup_{n=1}^{\infty} K_{n}=G$ then $\left(F_{n}\right)_{n=1}^{\infty}$ is a Følner sequence in $G$.

Proof. Let $R=\left(R_{g}\right)_{g \in G}$ be an ergodic measure preserving Bernoulli (free) action of $G$ on a standard probability space $(Y, \nu)$. Denote by $\mathcal{R}$ the $R$-orbit equivalence 
relation on $Y$. We also denote by $\widehat{\mathcal{R}}$ the orbit equivalence relation of the product $G$ action $T \times R:=\left(T_{g} \times R_{g}\right)_{g \in G}$. Since $G$ is amenable, $\mathcal{R}$ and $\widehat{\mathcal{R}}$ are both hyperfinite. Let $\left(\mathcal{R}_{n}\right)_{n=1}^{\infty}$ be a filtration of $\mathcal{R}$. For $y \in Y$ and $n>0$, let $G_{y, n}$ stand for the finite subset of $G$ such that $\mathcal{R}_{n}(y)=\left\{R_{g} y \mid g \in G_{y, n}\right\}$. For each $n>0$, we now define a finite equivalence relation $\widehat{\mathcal{R}}_{n}$ on $X \times Y$ by setting

$$
(x, y) \sim_{\widehat{\mathcal{R}}_{n}}\left(x^{\prime}, y^{\prime}\right) \quad \text { iff }\left(y, y^{\prime}\right) \in \mathcal{R}_{n} \text { and } x^{\prime}=T_{g} x
$$

for the only element $g \in G$ such that $y^{\prime}=T_{g} y$.

Then $\left(\widehat{\mathcal{R}}_{n}\right)_{n=1}^{\infty}$ is a filtration of $\widehat{\mathcal{R}}$. It follows from the martingale convergence theorem there there is a $(\mu \times \nu)$-conull subset $Z$ of $X \times Y$ such that

$$
\lim _{n \rightarrow \infty} E\left(f \otimes 1 \mid \operatorname{Inv}\left(\widehat{\mathcal{R}}_{n}\right)\right)(x, y)=E(f \otimes 1 \mid \operatorname{Inv}(\widehat{\mathcal{R}}))(x, y)
$$

for every $f \in \mathcal{L}$ at each $(x, y) \in Z$. We now note that

$$
\begin{aligned}
E\left(f \otimes 1 \mid \operatorname{Inv}\left(\widehat{\mathcal{R}}_{n}\right)\right)(x, y) & =\frac{\sum_{g \in G_{y, n}} f\left(T_{g} x\right) \frac{d \mu \circ T_{g}}{d \mu}(x)}{\sum_{g \in G_{y, n}} \frac{d \mu \circ T_{g}}{d \mu}(x)} \text { and } \\
E(f \otimes 1 \mid \operatorname{Inv}(\widehat{\mathcal{R}}))(x, y) & =E(f \mid \operatorname{Inv}(\mathcal{T}))(x) .
\end{aligned}
$$

By the Fubini theorem, there is $y_{0} \in Y$ and a $\mu$-conull subset $X_{0}$ in $X$ such that $X_{0} \times\left\{y_{0}\right\} \subset Z$. We let $F_{n}:=G_{y_{0}, n}$. Since $\mathcal{R}_{1}\left(y_{0}\right) \subset \mathcal{R}_{2}\left(y_{0}\right) \subset \cdots$ and $\bigcup_{n=1}^{\infty} \mathcal{R}_{n}\left(y_{0}\right)=\mathcal{R}\left(y_{0}\right)$, it follows that $F_{1} \subset F_{2} \subset \cdots$ and $\bigcup_{n=1}^{\infty} F_{n}=G$. It remains to substitute (A-3) into (A-2) to obtain (A-1).

By [Da, Lemma 2.2], for each $m>0$,

$$
\nu\left(\left\{y \in Y \mid G_{y, n} \text { is }\left[K_{m}, \epsilon_{m}\right] \text {-invariant }\right\}\right)>1-\epsilon_{m}
$$

eventually in $n$. Hence passing to a subsequence in $\left(\mathcal{R}_{n}\right)_{n=1}^{\infty}$ and applying the Borel-Cantelli lemma we can choose $y_{0}$ in such a way that $F_{n}$ is $\left[K_{m}, \epsilon_{m}\right]$-invariant for all $n \geq m>0$.

\section{REFERENCES}

[AlPi] D. J. Aldous, J. W. Pitman, On the zero-one law for exchangeable events, Ann. Probab. 7 (1979), 704-723.

[Da] A. I. Danilenko, Entropy theory from orbital point of view, Monatsh. Math. 134 (2001), 121-141.

[DaLe] A. I. Danilenko and M. Lemańczyk, K-property for Maharam extensions of nonsingular Bernoulli and Markov shifts, Ergod. Th. \& Dynam. Sys. (to appear).

[DaSi] A. I. Danilenko and C. E. Silva, Ergodic theory: non-singular transformations, Mathematics of Complexity and Dynamical Systems, Springer, New York, 2012, pp. 329-356.

[FeMo] J. Feldman and C. C. Moore, Ergodic equivalence relations, cohomology and von Neumann algebras, I, Trans. Amer. Math. Soc. 234 (1977), 289-324.

[GlWe] E. Glasner and B. Weiss, Weak mixing properties for non-singular actions, Ergod. Th. \& Dynam Sys. 36 (2016), 2203-2217.

[Ha] T. Hamachi, On a Bernoulli shift with non-identical factor measures, Ergod. Th. \& Dynam. Sys. 1 (1981), 273-284.

[Ho] M. Hochman, On the ratio ergodic theorem for group actions, J. London Math. Soc. 88 (2013), 465-482. 
[JaKeLo] S. Jackson, A. S. Kechris and A. Louveau, Countable Borel equivalence relations, Journal of Math. Logic 2 (2002), 1-80.

[Ka] S. Kakutani, On equivalence of infinite product measures, Ann. Math. 49 (1948), 214224.

[Ko1] Z. Kosloff, On a type III $I_{1}$ Bernoulli shift, Ergodic Theory \& Dyn. Syst. 31 (2011), $1727-1743$.

[Ko2] - The zero-type property and mixing of Bernoulli shifts, Ergodic Theory \& Dyn. Syst. 33 (2013), 549-559.

[Ko3] On the K property for Maharam extensions of Bernoulli shifts and a question of Krengel, Israel J. Math. 199 (2014), 485-506.

[Ko4] - Proving ergodicity via divergence of ergodic sums, Preprint, arXiv:1802.07780.

[Kr] U. Krengel, Transformations without finite invariant measure have strong generators, Lecture Notes in Math. 160 (1970), Springer, New York, 133-157.

[SiTh] C. E. Silva and P. Thieullen, A skew product entropy for nonsingular transformations, J. London Math. 52 (1995), 497-516.

[OrWe] D. Ornstein and B. Weiss, Entropy and isomorphism theorems for actions of amenable groups, J. Anal. Math. 48 (1987), 1-141.

[VaWa] S. Vaes and J. Wahl, Bernoulli actions of type III $I_{1}$ and $L^{2}$-cohomology, Geom. and Funct. Anal. 28 (2018), 518-562.

Institute for Low Temperature Physics \& Engineering of National Academy of Sciences of Ukraine, 47 Nauky Ave., Kharkiv, 61103, UKRaine

E-mail address: alexandre.danilenko@gmail.com 